\title{
Evaluation of miRNA detection methods for the analytical characteristics necessary for clinical utilization
}

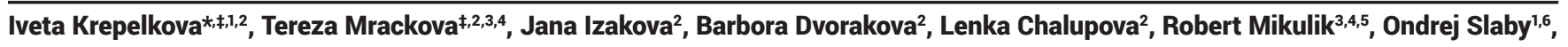
Milan Bartos ${ }^{2,7}$ \& Viktor Ruzicka²

\begin{abstract}
miRNAs are promising biomarkers but methods for their measurement are not clear. We therefore examined three miRNA detection technologies and considered the analytical characteristics essential for clinical utilization. TaqMan assays, SplintR-qPCR and miREIA were compared for their absolute quantification bias, conformity and robustness. Absolute concentrations of miR-142-5p, miR-23a-3p and miR-93-5p were measured with all three methods using 30 samples. Robustness was evaluated by measurement of miR-21-5p in five uniform experiments. Correlations were miRNA-specific, but we observed a different absolute concentration range in RT-qPCR ( $\mathrm{fmol} / \mu \mathrm{l}$ ) and methods evading the RT process $(\mathrm{amol} / \mu \mathrm{l})$. Consistently, RT-less methods reported better robustness (CV 8-19\%) than RT-qPCR (CV 39-50\%). The calibration curve in TaqMan Advanced assay was influenced by dilution media. Methods avoiding RT seem to be a promising future alternative for miRNA measurement.
\end{abstract}

\section{METHOD SUMMARY}

Three miRNA detection technologies were compared: 1) RT-qPCR where the RT step was performed with either a specific (TaqMan miRNA assay) or universal (TaqMan Advanced assay) priming strategy; 2) miREIA technology, using hybridization and specific antibody to DNA/RNA hybrids and 3) SplintR-qPCR, which utilizes a hybridization and ligation step followed by qPCR.

\section{KEYWORDS:}

miREIA - miRNA biomarkers - miRNA enzyme immunoassay • RT-qPCR - SplintR-qPCR

${ }^{1}$ Central European Institute of Technology, Masaryk University, Brno, Czech Republic; ${ }^{2}$ BioVendor - Laboratorní medicína a.s., Brno, Czech Republic; ${ }^{3}$ Faculty of Medicine, Masaryk University, Brno, Czech Republic; ${ }^{4}$ International Clinical Research Center (FNUSA-ICRC), Brno, Czech Republic, ${ }^{5}$ Department of Neurology, St Anne's University Hospital, Brno, Czech Republic; ${ }^{6}$ Masaryk Memorial Cancer Institute, Department of Comprehensive Cancer Care, Brno, Czech Republic; ${ }^{7}$ Department of Experimental Biology, Faculty of Science, Masaryk University, Brno, Czech Republic; *Author for correspondence: krepelkova@biovendor.com ¥These authors contributed equally

BioTechniques 66: 277-284 (June 2019) 10.2144/btn-2019-0021
miRNAs are small noncoding RNA molecules that play an important regulatory role in gene translation through silencing or degradation of target mRNAs [1]. They influence a wide range of major biological processes including differentiation, proliferation, metabolism, apoptosis or inflammation [2-5]. Deregulation of miRNA levels within cells is associated with various diseases, so they have an immense potential to serve as diagnostic or prognostic biomarkers [6]. Utilization of circulating miRNA as a noninvasive diagnostic tool in liquid biopsies is also very promising [7].

However, precise detection and quantification of miRNA is challenging and limits further miRNA research. Therefore, there is a need for a reliable miRNA detection platform that could be used routinely in laboratories. Problematic determination of miRNAs in the sample is caused by the short length of these molecules (approximately 22 nucleotides), small sequence differences between miRNAs and also their low concentrations in body fluids [8]. Nevertheless, many approaches for detecting miRNA have been introduced. The most common are RNA sequencing (RNA-Seq), microarrays and reverse transcription PCRs (RT-qPCR) [9].

RNA-Seq utilizes NGS for highthroughput analysis of miRNA. The most widespread NGS technology is based on individual binding of fluorescent-labeled nucleotides. This approach can determine many heterogeneous samples in one run and simultaneously provides sequence information for each
miRNA contained in the sample. NGS, as the only method, can be used to discover new miRNA molecules and is also the most sensitive principle for miRNA identification and quantification. On the other hand, NGS is very time-consuming, because extensive processing steps are required to convert a sample into a library for sequencing. High volume of sample is consumed in this process and sophisticated software is required for evaluating results. The combination of expensive chemistry and instrumentation means a high cost per analysis [10].

Microarray is used for multiplex analysis of already described miRNAs. The central concept is based on hybridization of the target with a specific probe to measure the abundance of nucleic acid sequences through fluorescencebased detection [11]. Many different probes are usually bound on a solid surface and thus miRNA expression chips with hundreds of targets are created. Chips are commercially available with preselected targets or can be tailored to the customer's needs [9]. In principle, microarrays can provide very precise information about absolute concentration of miRNA. However, in practice, there is huge quantification bias due to unreliable background corrections and normalization, so they are usually considered for relative quantification only. The sensitivity of microarray is lower than RNA-seq because there is no amplification step. Measurement is also limited by the sensitivity of the fluorescence reader, so expensive instrumentation is required $[9,11]$. 
- NGS and microarrays together are probably not suitable for routine utilization; their strength lies in the discovery of new miRNAs and the search for differential miRNA expression connected to pathological conditions. The one with the biggest potential for diagnostic purposes is the gold-standard RT-qPCR [9].

For detection of short RNA molecules (including miRNA), adaptions of the standard RT-qPCR techniques have been established and they are currently the most widely used [12]. PCR is preceded by enzymatic transformation of mature miRNA into a cDNA sequence and then amplification of this sequence by appropriate primers [13]. The most error-prone step of the process is the RT reaction, which can be performed in two ways. The first method uses specific stem-loop RT primers to produce cDNA from specific miRNA. The primer hybridizes to the $3^{\prime}$ section of the miRNA while at the same time prolonging the $3^{\prime}$ end of the molecule with its $5^{\prime}$ end to generate a nicked RNA hybrid of miRNA and stem-loop primer. After RT, the resulting cDNA is amplified by qPCR using a forward primer specific to the miRNA sequence and a reverse primer specific to the stem-loop primer $[14,15]$. The second method extends the miRNA at its $3^{\prime}$ end with a poly $(A)$ tail by using enzyme $\operatorname{poly}(A)$ polymerase. The resulting polyadenylated RNA is then reverse transcribed using a universal oligo(dT) primer, therefore all miRNAs within the RNA sample are transcribed $[12,14,16,17]$. Following measurement of selected miRNAs, specific PCR primers are used for amplification of reverse transcribed cDNA $[14,17]$.

As RT-qPCR techniques can be performed on relatively standard equipment, these tests are widely available and can be automated. Sensitivity, specificity and reproducibility of RT-qPCR is generally considered as very good, but an undeniable bias of the RT process is often overlooked. This technology is useable only for molecules that have been previously identified, but despite its imperfections in comparison with the above-mentioned techniques it is the most attractive one for routine testing in clinical laboratories [14].

Nevertheless, other promising approaches for miRNA detection have recently been presented. The first approach preserves qPCR as the visualization system but innovates the process of cDNA synthesis. RT is replaced by enzymatic ligation of two DNA oligonucleotides splinted by target miRNA. Because ligation of single-strand breaks in DNA/RNA hybrids is not usual in living organisms, success of this technology consists requires a suitable ligation enzyme. Only recently, Chlorella virus DNA ligase (SplintR ligase) has been found, which is able to catalyze such a reaction with sufficient efficacy for miRNA detection. Multiple miRNAs have already been determined by SplintR-qPCR using a double-

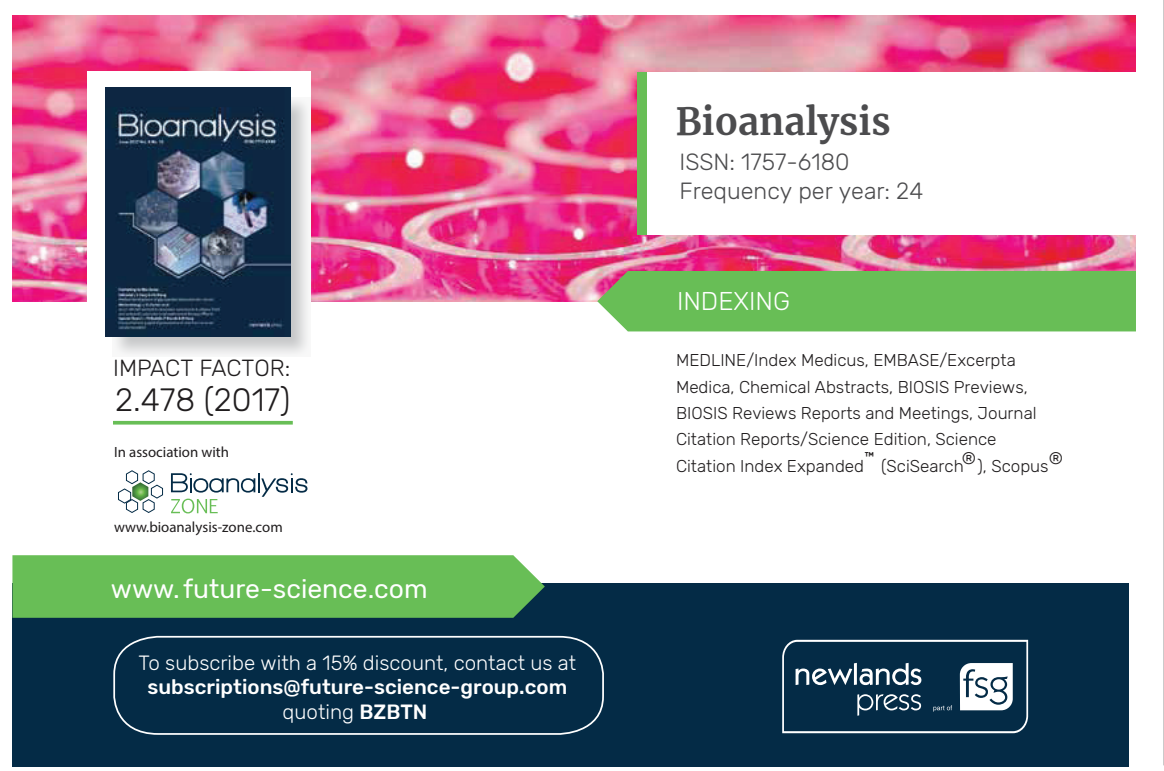

quenched TaqMan probe. Only a 4-6 bp overlap between a DNA probe and miRNA was required for efficient ligation, providing more flexibility in probe design and allowing it to reach higher specificity [18].

The second approach utilizes the properties of a unique monoclonal antibody specific only to perfectly matched DNA/RNA hybrids. This system was introduced in 2015 by Kappel et al. and later was transformed to the format of a classical immunoassay named miREIA (miRNA enzyme immunoassay) [19]. This method does not involve $\mathrm{RT}$ nor amplification. The protocol is based on hybridization of a DNA probe to the miRNA target and followed by the subsequent quantification of generated DNA/ RNA hybrids. The workflow is very similar to the well-known ELISA principle, which is convenient for clinical laboratory settings. Moreover, this system is also applicable for standard immunoanalyzers and allows for multiplex measurement [19].

Although the technologies mentioned above could provide solutions for miRNA measurement, there is still a need for more data focused on their analytical characteristics. In this study we compared RT-qPCR, SplintR-qPCR and miREIA detection methods for their analytical characteristics necessary for clinical utilization.

\section{MATERIALS \& METHODS}

Synthetic oligonucleotides

RNA oligonucleotides and DNA primers were synthetized by Eurofins genomics. Sequences of the miRNA oligonucleotides were obtained from miRbase Release 21 (www.mirbase.org).

Probes \& primers sequences for

SplintR-qPCR

Probe A23: 5' CCTCTCTATGGGCAGTCGGTGAGGAAATCCCT $3^{\prime}$

Probe B23: 5' PHO-GGCAATGTGATTGAGTCGGGAGACACGCAGGG 3'

Probe A93: 5' CCTCTCTATGGGCAGTCGGTGACTACCTGCACG 3'

Probe B93: 5' PHO-AACAGCACTTTGTGAGTCGGGAGACACGCAGGG 3'

Probe A142: 5' CCTCTCTATGGGCAGTCGGTGACGTAGTGCTT 3'

Probe B142: 5' PHO-TCTACTTTATGTGAGTCGGGAGACACGCAGGG 3'

Probe A21: 5' CCTCTCTATGGGCAGTCGGTGATCAACATCAG 3' 
Probe B21: 5' PHO-TCTGATAAGCTATGAGTCGGGAGACACGCAGGG $3^{\prime}$

Forward primer: 5' GCTCGACCTCTCTATGGGC 3'

Reverse primer: $5^{\prime}$ TTAAGCCCTGCGTGTCTCC 3'

\section{Samples \& RNA isolation}

A total of 19 samples of whole blood and 11 samples of peripheral blood mononuclear cells (PBMCs) were used in this study. All 30 samples were provided by healthy volunteers. $2.5 \mathrm{ml}$ of whole blood was collected in PAXgene Blood RNA Tubes (Preanalytics) and handled according to manufacturer's instructions. For obtaining the PBMC layer, $5 \mathrm{ml}$ of blood was collected in EDTA tubes (Vacutainer System BD) and overlaid to $10 \mathrm{ml}$ of Histopaque (Sigma Aldrich). PBMCs were then separated by gradient centrifugation.

Total RNA including miRNA was purified manually with miRNeasy Mini Kit (Qiagen). Briefly, tubes were thawed for $2 \mathrm{~h}$ at room temperature and then centrifuged for $10 \mathrm{~min}$ at $4500 \mathrm{xg}$. The supernatant was removed immediately, and the pellets were resuspended in $4 \mathrm{ml}$ RNase-free water and centrifugated again. $500 \mu \mathrm{l}$ of QIAzol solution (Qiagen) was added to obtained pellets. Then, the isolation proceeded according to the manufacturer's recommendations. The total elution volume was $50 \mu \mathrm{l}$ of RNAse-free $\mathrm{H}_{2} \mathrm{O}$. Quality of isolated RNA was determined using Nanodrop ND-2000 (ThermoFisher Scientific). The ratio of absorbance at $260 / 280 \mathrm{~nm}$ was 1.95-2.13 for all samples. Isolated RNA was stored at $-80^{\circ} \mathrm{C}$ until use.

\section{miRNA detection}

RT-qPCR was performed using TaqMan miRNA assays or TaqMan Advanced assays (both Thermofisher Scientific) according to manufacturer's instructions. $10 \mathrm{ng}$ of total RNA was used as an input amount for CDNA synthesis. All calibration curves were done using synthetic RNA, by ten-times serial dilutions of the RT product. Reactions were performed in CFX96 real-time detection system (BioRad) and results were evaluated with related software (BioRad).

The SplintR-qPCR procedure was obtained from Jin et al. [18] and modified in-house. The hybridization mix was composed of: $1 \mu \mathrm{l}$ of probe $\mathrm{A}(1 \mathrm{pmol} / \mu \mathrm{l})$; $1 \mu \mathrm{l}$ of probe $B(1 \mathrm{pmol} / \mu \mathrm{l}), 2 \mu \mathrm{l}$ of hybridization buffer ( $1 \%$ triton X-100, 10\% BSA, $50 \%$ tris-buffered saline), $0.02 \mu$ of RNAse inhibitor (Sigma Aldrich) and $4.98 \mu \mathrm{l}$ of nuclease-free water. $1 \mu \mathrm{l}$ of undiluted sample was added to the total reaction volume of $10 \mu \mathrm{l}$. Hybridization reactions were incubated for $3 \mathrm{~min}$ at $4^{\circ} \mathrm{C}, 2 \mathrm{~min}$ at $85^{\circ} \mathrm{C}$ and $5 \mathrm{~min}$ at $37^{\circ} \mathrm{C}$. The ligation step was performed using $1 \mu$ l of SplintR ligase (New England Biolabs), $2 \mu$ of ligation buffer (New England Biolabs) and $15 \mu \mathrm{l}$ of nuclease-free water. $2 \mu \mathrm{l}$ of hybridization product was added to the total volume of $20 \mu \mathrm{l}$. Tubes were incubated for $15 \mathrm{~min}$ at $25^{\circ} \mathrm{C}, 5 \mathrm{~min}$ at $4^{\circ} \mathrm{C}$, and $10 \mathrm{~min}$ at $65^{\circ} \mathrm{C}$ to ligate the product and then held at $4^{\circ} \mathrm{C}$. qPCR was performed in the final volume of $20 \mu$ l using $0.5 \mu$ l of forward and reverse primer $(10 \mu \mathrm{M}), 10 \mu \mathrm{l}$ of IQ Syber Green supermix (BioRad), $7 \mu$ l of nuclease-free water and $2 \mu$ ligation product (ten-times diluted). The qPCR protocol was as follows: $95^{\circ} \mathrm{C}$ for 3 min and 45 cycles of $95^{\circ} \mathrm{C}$ for 10 $\mathrm{s}, 55^{\circ} \mathrm{C}$ for $20 \mathrm{~s}$ and $72^{\circ} \mathrm{C}$ for $1 \mathrm{~s}$. Reaction specificity was assessed by melting curve analysis immediately after the qPCR. All incubation and amplification steps were done in the CFX96 real-time detection system (BioRad). Results were evaluated with related software. All calibration curves were constructed by ten-times serial dilution of ligation product.

miREIA (BioVendor - Laboratorní medicína) measurement was done according to manufacturer's instructions. Samples were diluted at least five times. All calibration curves were constructed according to the product data sheet. Hybridization was done in peqStar thermocycler (Peqlab). All reactions were incubated in Incucell (BMT) incubator and absorbance was measured in a 96-well reader EL808 (Biotek). Sample concentrations were calculated with GEN software.

\section{Analytical characteristics of miRNA detection methods}

Correlation of TaqMan miRNA assays, SplintR-qPCR and miREIA was determined for miR-142-5p, miR-23a-3p and miR-93-5p. Absolute concentrations of all miRNA targets were measured in 19 whole-blood and 11 PBMC samples. Synthetic miRNAs were used to generate standard curves. All experiments were done in duplicate.

Influence of different dilution media for standard curve preparation was evaluated for miR-142-5p. Synthetic RNA was diluted in nuclease-free water, a cocktail of nonsense miRNAs $(1000 \mathrm{fmol} /$ $\mu \mathrm{l}$; miR-208a-3p, miR-376c-3p, miR-126-5p, miR-197-3p, miR-222-3p) and yeast RNA ( $5 \mathrm{ng} / \mu \mathrm{l}$, Thermofisher Scientific). For the TaqMan miRNA and TaqMan Advanced assays, synthetic RNA was diluted to obtain a final concentration of miR-142-5p $1000 \mathrm{fmol} / \mu \mathrm{l}$ and $7 \mathrm{ng}$ of total RNA was used as an input amount. Hybridization is not prone to changes in the total RNA amount, so nonsense miRNA and yeast RNA was used instead of water solvent in SplintR-qPCR and miREIA protocols. Starting concentration for SplintR-qPCR was $100 \mathrm{fmol} / \mu \mathrm{l}$ and for miREIA $12.5 \mathrm{amol} /$ $\mu$ l of synthetic miR-142-5p. Shifts in Cq and absorbance values, respectively, were evaluated between different dilution media.

Robustness of the methods (TaqMan miRNA assays, SplintR-qPCR and miREIA) was assessed in one whole-blood and one PBMC RNA isolate. Samples were aliquoted and stored at $-80^{\circ} \mathrm{C}$ to avoid repeated freezing-thawing cycles. Absolute concentration of miR-21-5p was determined using standard curve. Results obtained by uniform measurement over 5 distinct days were used to calculate the $\mathrm{CV}$ for each sample. Each measurement was done in two replicates. $\mathrm{CV}$ was calculated according to the formula:

$$
C V=\frac{S D c}{\varnothing c}
$$

(SDc: Standard deviation of concentration values; Øc: Average of concentration values)

\section{RESULTS \& DISCUSSION}

The mandatory requirement of the EU for use of any type of diagnostic test in the routine lab is CE-IVD certification. In this process, predefined analytical characteristics (including robustness, specificity, sensitivity, etc.) of the diagnostic test have to meet some expected criteria to pass the certification. This rule will not avoid the oncoming clinical use of miRNA molecules.

It is important to realize that the demanding characteristics of the CE-IVD test are influenced not only by the power of the biomarker itself, but also by the 


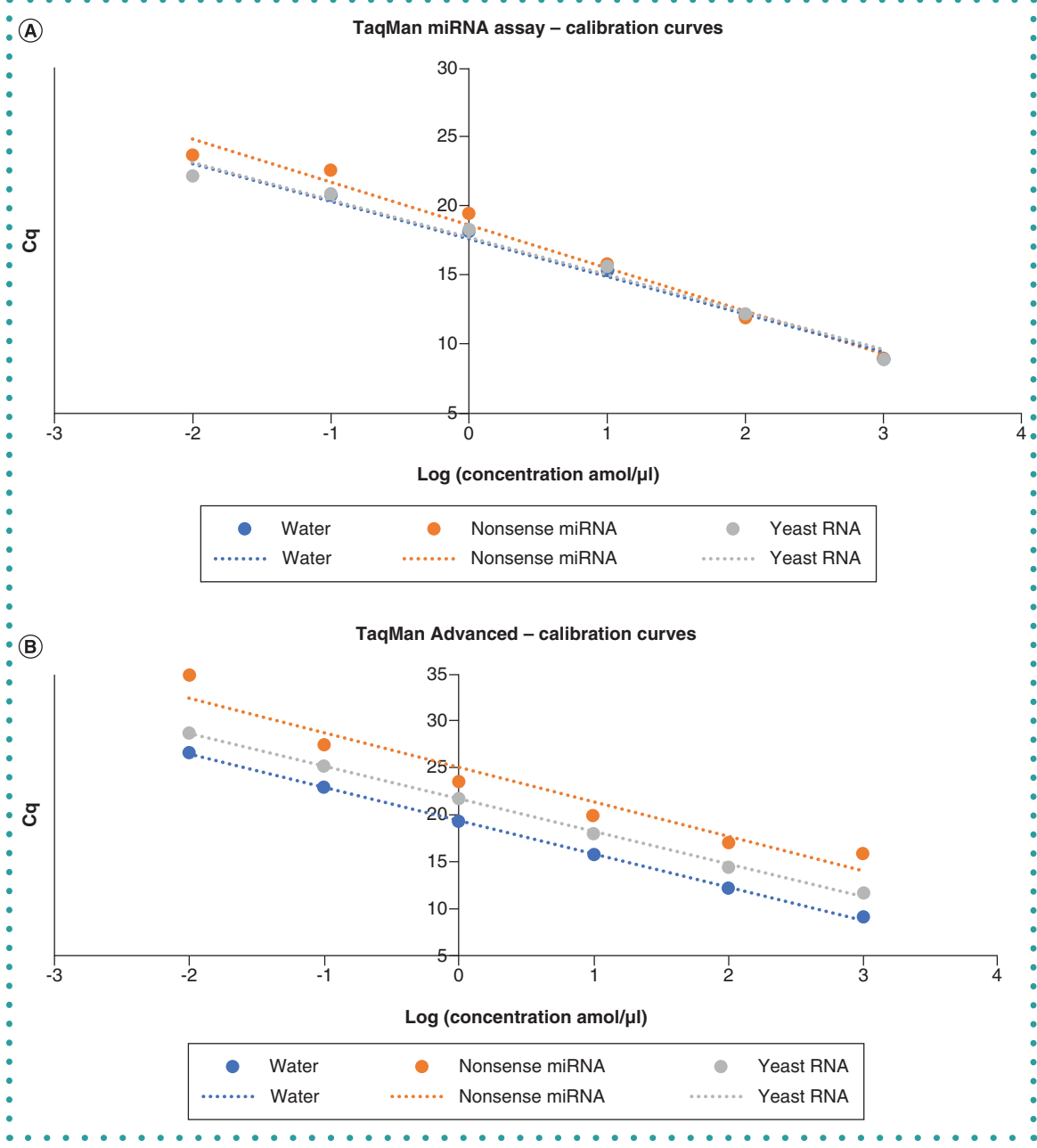

Figure 1. Comparison of synthetic calibration curves of miR-142-5p dissolved in different dilution media (blue - water, orange - nonsense miRNA, grey - yeast RNA). Measured by (A) RT-qPCR, (B) RT-qPCR ADVANCED, (C) SplintR-qPCR and (D) miREIA.

> measurement technology chosen for biomarker detection. Contrary to this, especially in world of miRNA molecules, this issue does not pay much attention and necessary analytical characteristics of miRNA detection methods are not being tested. Therefore, we evaluated three miRNA detection technologies that are based on different principles: (1) well-known RT-qPCR, RT step is based on using specific (TaqMan miRNA assay) or universal (TaqMan ADVANCED assay) priming strategy; (2)miREIA technology, using hybridization and specific antibody to DNA/RNA hybrids [19]; (3)SplintR-qPCR, a combination of the previously mentioned methods, which utilizes a hybridization and ligation step followed by qPCR [18].

There are many studies that focus on the sensitivity and specificity of RT-qPCR methods. Generally, using a specific priming strategy is usually more specific, but less sensitive. By contrast, the universal RT priming strategy is more sensitive, but generates higher background [20]. Little information regarding specificity and sensitivity is available regarding miREIA and SplintR-qPCR because they are new and less common. Based on our experiments we can claim that these methods are less sensitive than RT-qPCR. In most cases, we were not able to detect miRNA in serum or plasma samples (data not shown). Another disadvantage of hybridization-based methods is their lower dynamic range and for miREIA technology there is also higher sample consumption. However, in clinical applications it is also necessary to evaluate another important characteristic of the assays absolute quantification, which makes it possible to compare results obtained by different laboratories, and also robustness and accuracy of the methods. Therefore, those performance parameters were investigated in this study.

\section{Influence of dilution media on synthetic calibration curves}

The construction of a proper calibration curve is the first step for reliable absolute quantification, but this remains a challenging topic in RT-qPCR methods. The most crucial step is to ensure the same efficiency of RT in the sample and the synthetic material used for generating the calibration curve. Usually, some background RNA is recommended to dissolve the standard material [21]. We wanted to examine how crucial it is in our miRNA detection methods.

The influence of background RNA on the miRNA standard curve preparation was evaluated by serial dilution of synthetic miR-142-5p in different dilution media. We used yeast RNA, which is recommended by the MIQE guidelines [22], cocktail of nonsense miRNA to mimic isolation only short RNA from the sample and compared it with the miRNA dissolved in nuclease-free water, which is the most commonly used solvent. We observed no significant media influence in singleplex RT-qPCR (Figure 1A) nor in methods without RT (miREIA and SplintR-qPCR) (Figure 1C \& D). On the other hand, we have shown a significant $\mathrm{Cq}$ shift in the calibration curves determined by TaqMan Advanced qPCR method (Figure 1B). The average $C q$ shift was 5.6 between the same concentration of synthetic RNA dissolved in water and a cocktail of nonsense miRNA and 2.3 between water and yeast RNA. As a consequence, different preparations of the synthetic calibration curve with this method could dramatically influence the absolute concentration of miRNA in the sample.

TaqMan Advanced assay utilizes the universal conversion of all miRNA in the sample. In all cases, the same amount of total RNA and the same concentration of standard RNA material was used for RT, so the requirements for RT were maintained. However, different total amounts of standard miRNA were present in the sample. When assuming the same efficiency of universal primers and reverse transcriptase in the $\mathrm{RT}$ reaction, a different amount of standard miRNA can be transformed to the form of cDNA, which could 
cause Cq shift [23]. This universal RT principle used in the TaqMan Advanced assay was therefore inappropriate for calibration curve preparation and for that reason was excluded from the correlation and robustness experiments.

\section{Correlation of methods}

Correlation and absolute concentration experiments were determined for TaqMan miRNA assay, SplintR-qPCR and miREIA. 30 RNA isolates from whole blood and PBMCs were measured for three miRNA targets - miR-142-5p, miR-23a-3p and miR-93-5p. Those targets were published previously as important candidate markers for early diagnosis of colorectal cancer [24].

Individual correlation patterns were miRNA- and assay-specific. For miR-142-5p we found a strong correlation between SplintR-qPCR and miREIA $\left(R^{2}=0.883\right.$; Figure $2 A$ ) and marginal correlation between RT-qPCR and the other methods $\left(R^{2}=0.501\right.$ and 0.493; Figure 2B \& $\left.C\right)$. A similar pattern was also observed for miR-93-5p. A very high correlation coefficient was found between SplintRqPCR and miREIA ( $R^{2}=0.792$; Figure $\left.2 D\right)$ and also for SplintR-qPCR and RT-qPCR $\left(R^{2}=0.699\right.$; Figure $\left.2 E\right)$ and lower correlation for miREIA and RT-qPCR $\left(\mathrm{R}^{2}=0.567\right.$; Figure 2F). A different pattern was shown for miR-23a-3p. Good correlation was observed for SplintR-qPCR and RT-qPCR $\left(R^{2}=0.686\right.$; Figure $\left.2 \mathrm{H}\right)$, but only weak correlation of miREIA with either QPCR methods $\left(R^{2}=0.236\right.$ and 0.231 ; Figure $\left.2 G \& I\right)$ was found.

Despite correlations, the most problematic miRNA was miR-23a-3p. Unlike miR-142-5p and miR-93-5p, this miR-23a-3p is a member of a larger family that also includes miR-23b and miR-23c ( $w w w$. mirbase.org). Measurement of a miRNA that has another sibling is always more complicated in terms of specificity [25]. This could cause greater variation in measurements by different technologies and, as a consequence, lower correlation results [26]. Measurement of miR-142-5p, miR-93-5p and also miR-21-5p (Table 1) supports the previously indicated fact that the RT step causes most of the variability produced in RT-qPCR [27], and this variability is higher with RNA with a shorter length of template [28]. Although

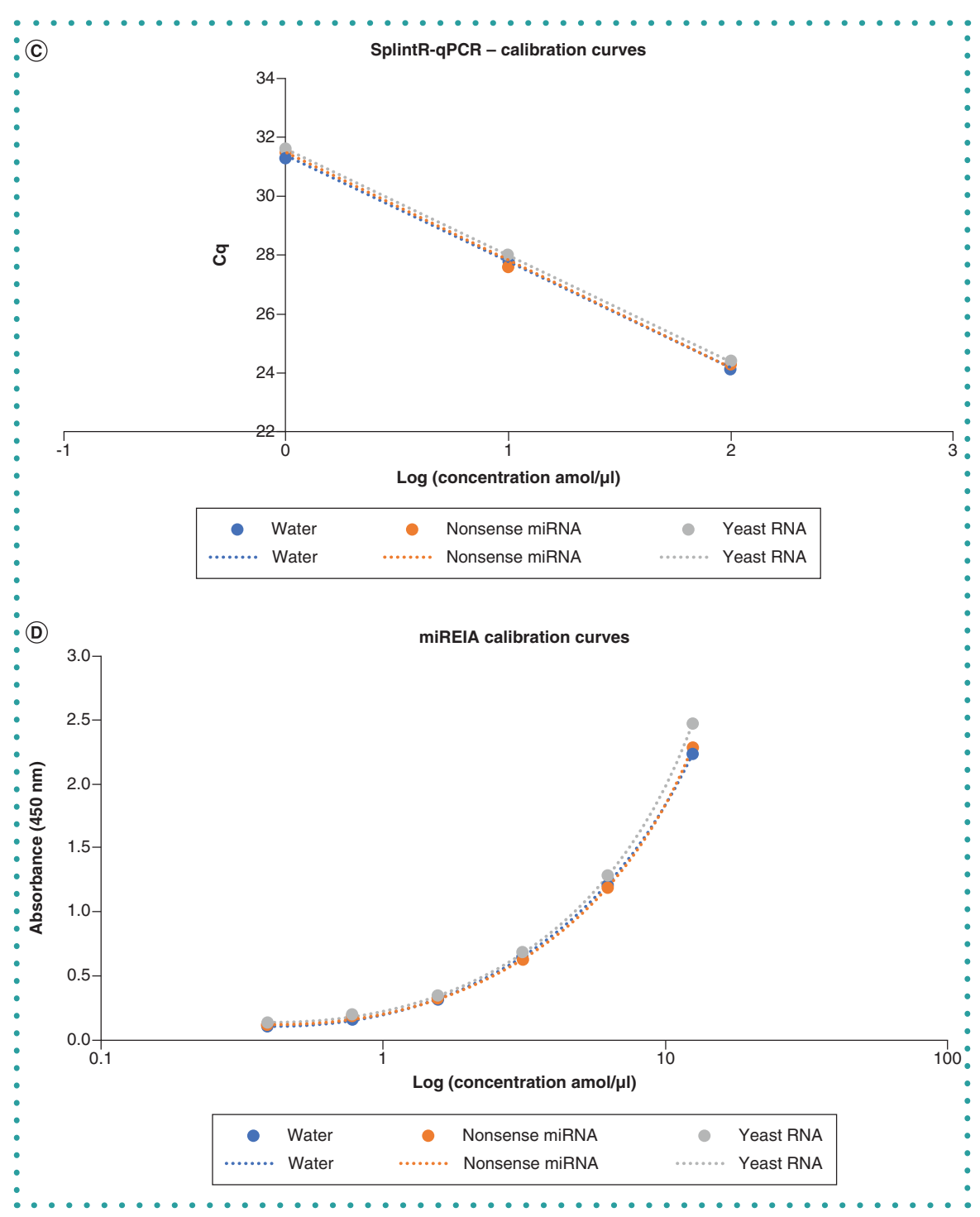

Figure 1 (cont.). Comparison of synthetic calibration curves of miR-142-5p dissolved in different dilution media (blue - water, orange - nonsense miRNA, grey - yeast RNA). Measured by (A) RT-qPCR, (B) RT-qPCR ADVANCED, (C) SplintR-qPCR and (D) miREIA.

RT-qPCR is the most commonly used technology in research [23], results are usually evaluated as relative difference between two compared groups. Surprisingly, only a minority of studies have focused on absolute quantification of miRNA by RT-qPCR [29-31]. Unfortunately, we were not able to compare our data with these independent studies.

\section{Determination of absolute \\ concentration}

Generally, we observed absolute concentration conformity in the methods that evaded the RT process and three logs shift in the concentrations measured by RT-qPCR; determined concentration by
RT-qPCR was approximately 1000-times higher. This shift was approximately constant across all measured targets and samples. One possible cause of the observed phenomenon is the RT step itself. $\mathrm{RT}$ is generally considered to be the most problematic step in RT-qPCR [32]. The efficiency of conversion of miRNA to cDNA strongly depends on the input amount of RNA, priming strategy $[27,33]$, template concentrations and background RNA in the sample [34]. Influence of these factors can be reduced by careful assay design. However, miRNA molecules are only 19-23 nucleotides long and very similar in sequence, making the specific RT-primer design very complicated [8]. This finding 
Table 1. Robustness of RT-qPCR, SplintR-qPCR and miREIA. miR-21-5p was determined in two samples on 5 distinct days.

\begin{tabular}{|c|c|c|c|c|c|c|c|c|c|}
\hline & \multicolumn{3}{|c|}{ RT-qPCR } & \multicolumn{3}{|c|}{ SplintR-qPCR } & \multicolumn{3}{|c|}{ MiREIA } \\
\hline & $\begin{array}{l}\text { Concentration } \\
\text { (fmol/ } \mu \mathrm{l})\end{array}$ & SD & CV (\%) & $\begin{array}{l}\text { Concentration } \\
\text { (amol/ul) }\end{array}$ & SD & CV (\%) & $\begin{array}{l}\text { Concentration } \\
\text { (amol/ } \mu \mathrm{l})\end{array}$ & SD & CV (\%) \\
\hline Whole blood & 7.4 & 2.9 & 39 & 19.1 & 3.6 & 19 & 18.2 & 3.1 & 17 \\
\hline Peripheral blood mononuclear cell & 10.2 & 5.1 & 50 & 64.8 & 10.4 & 16 & 54.1 & 4.2 & 8 \\
\hline
\end{tabular}

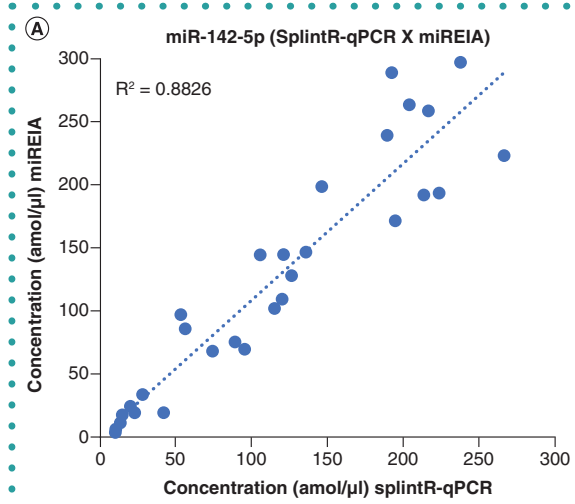

(C) miR-142-5p (miREIA X TaqMan)

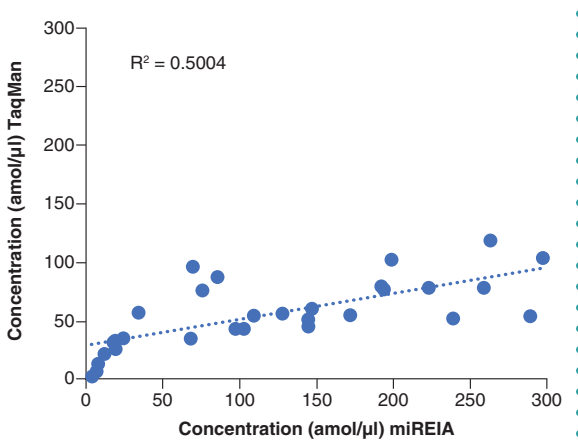

(B)
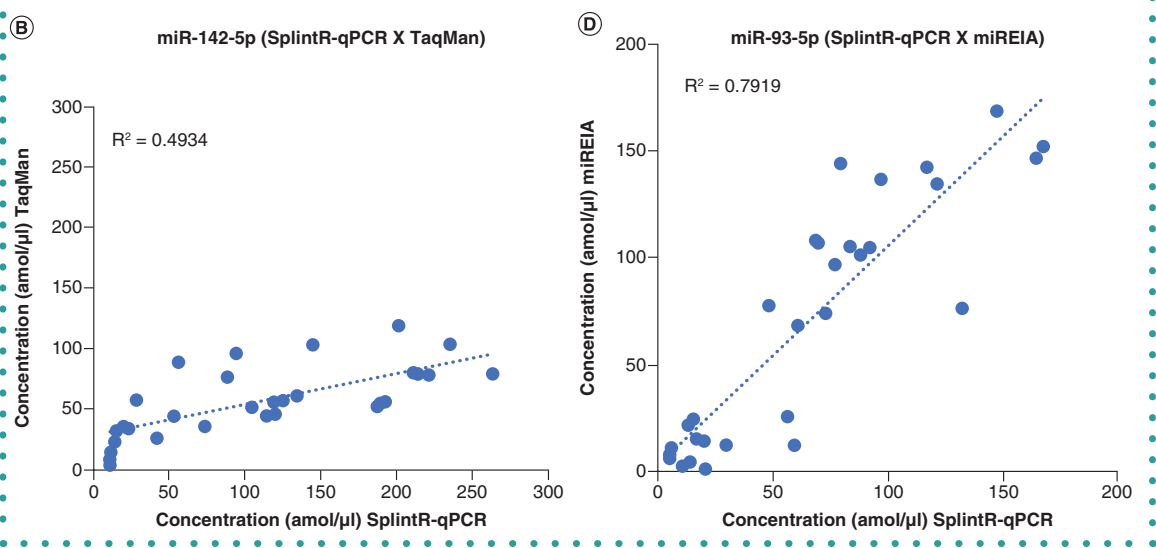

Figure 2. Correlations of absolute concentrations determined in 30 samples by TaqMan miRNA assays, SplintR-qPCR and miREIA. (A, B, C) miR-142-5p, (D, E, F) miR-93-5p and (G, H, I) miR-23a-3p.

> favors methods evading the RT step and supports the hypothesis that hybridization of a specific probe to miRNA is less sensitive to concentration changes of total RNA, or the presence of organic contaminants.

\section{Robustness}

To evaluate robustness of absolute quantification of RT-qPCR, SplintR-qPCR and miREIA we used two samples and measured the absolute concentration of miR-21-5p over 5 days. Results are shown in Table 1. The best robustness was observed for miREIA technology; CV was determined to be 17 and $8 \%$ for whole-blood and PBMC samples, respectively. Very similar results were obtained for SplintRqPCR (CV $=19$ and 16\%, respectively). Using these two methods we also reached accordance in absolute concentration of miR-21-5p. RT-qPCR exhibited the worst robustness of the methods tested: $\mathrm{CV}$ was determined to be 39 and $50 \%$ for wholeblood and PBMC samples, respectively. Also, the concentration of miR-21-5p determined by RT-qPCR was 1000 times higher than values determined by the other methods (as discussed above).

Using methods evading RT turned out to be beneficial for the robustness of the assay. RT has been reported to be a highly variable step in the RT-qPCR procedure [27].
On the other hand, this method has also been reported to be very robust with low CVs [35]. In this case we have to consider that the results were generated in Cq units and these units are logarithmic, not linear. If the $\mathrm{CV}$ is calculated based on the levels of Cq values, it will be much higher in the level of absolute concentration. These results are consistent with data published previously, where CVs of TaqMan miRNA and Exiqon assays were determined based on three independent measurements [30]. CV for miR-21-5p was determined to be 8.15 to $33.66 \%$, and CVs for other miRNA targets varied from 10 to $60 \%$ [29]. We think this is serious problem. Previous studies have reported significant differences in relative miRNA expression of as low as 1.5 -fold [36-38], but our findings suggest that such small differences in expression should be treated with caution because of a large deviation in repeated measurements [29]. Even if these differences were measured reliably, due to the high CV observed with the qPCR method it is not possible to clinically validate these small differences detected by RT-qPCR technology. With this last point we can see the biggest advantage of miREIA technology. It is very similar to ELISA kits that are commonly used and also has very similar properties, including robustness, making it likely to meet the demanding criteria of clinical validation.

\section{CONCLUSION}

In the last few decades, miRNA molecules dramatically increased their potential to serve as routine laboratory biomarkers due in part to the increasing number of clinical studies. However, miRNAs can only reach their full potential if the detection procedures are properly characterized. In our study, we evaluated methods of miRNA detection for analytical characteristics necessary for clinical utilization. Based on our results, RT-qPCR is the least appropriate. On the 
other hand, it remains the most sensitive method and definitely has a place in miRNA measurement. SplintR-qPCR and miREIA technologies are more robust and absolute quantification is more precise. They are suitable for clinical laboratories measuring samples from tissues and whole blood, but there has to be some development work carried out in terms of sensitivity, because plasma and serum samples are still the most common sample types being used.

\section{FUTURE PERSPECTIVE}

miRNAs and their detection and measurement are currently one of the most discussed topics across the scientific community, so a future increase in number of studies - including clinical ones focused on miRNA is highly probable. It is inevitable that there will be development of correct and reliable methods for miRNA measurement. In the near future there will be a great effort to move miRNA biomarkers into clinical practice, so the methods will be optimized according to clinical laboratory requirements. We expect increased attention to the field of analytical performance of current detection technologies.

\section{AUTHOR CONTRIBUTIONS}

IK and TM conceived the study, designed experiments and drafted the manuscript; both contributed equally. JI designed the miREIA assays used in the study. BD was responsible for sample collection and RNA isolation. LC helped with RT-qPCR measurement. MB was the main mentor and technical lead of the study. RM, OS and VR contributed to the manuscript revision.

\section{ACKNOWLEDGMENTS}

The authors would like to thank Brad Worden for language correction.

\section{FINANCIAL \& COMPETING INTERESTS DISCLOSURE}

This work has been supported by the European Regional Development Fund Project INBIO (No. CZ.02.1.01/0.0/0.0/16_0 26/0008451), by the project no. LQ1605 from the National Program of Sustainability II (MEYS CR) and by the project FNUSAICRC no. CZ.1.05/1.1.00/02.0123 (OP VaVpl). This project has received funding from the European Union's Horizon 2020 research and innovation programme under the Marie

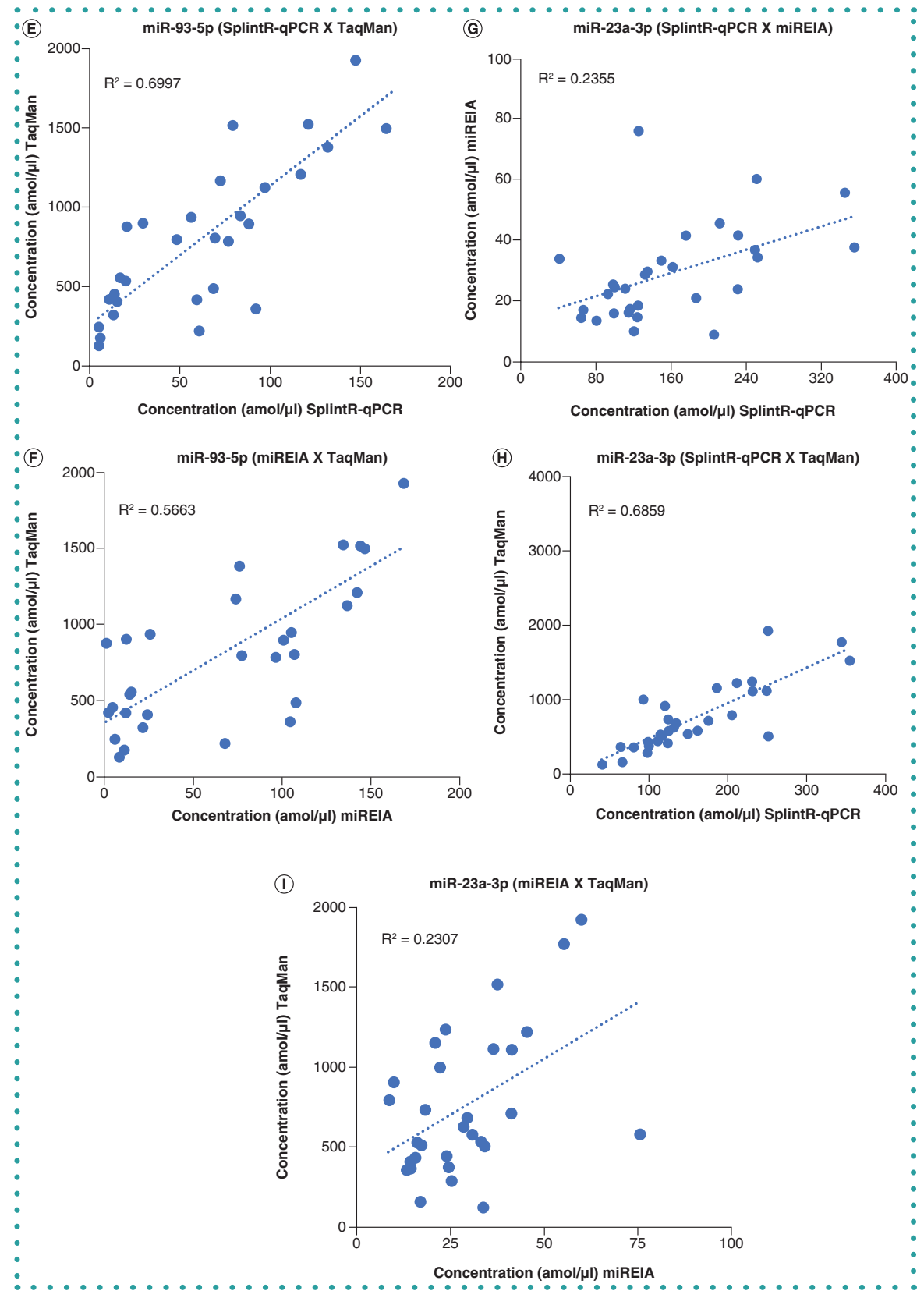

Figure 2 (cont.). Correlations of absolute concentrations determined in 30 samples by TaqMan miRNA assays, SplintR qPCR and miREIA. (A, B, C) miR-142-5p, (D, E, F) miR-93-5p and (G, H, I) miR-23a-3p.

Skłodowska-Curie grant agreement No 824036. This work has been supported by the Smart Accelerator project in the South Moravian Region JMK 27252/2017 - 4. ACTIVITY - ASSISTANCE number 047021/17/ORR - Building expertise for testing of biomarkers for early detection of cerebrovascular events. This work was also supported by the company BioVendor Laboratorní medicína. The authors have no other relevant affiliations or financial involvement with any organization or entity with a financial interest in or financial conflict with the subject matter or materials discussed in the manuscript apart from those disclosed.

No writing assistance was utilized in the production of this manuscript.

\section{OPEN ACCESS}

This work is licensed under the Creative Commons Attribution 4.0 License. To view a copy of this license, visit http://creativecommons.org/licenses/by/4.0/ 


\section{REFERENCES}

1. O'Brien J, Hayder H, Zayed Y, Peng C. Overview of microRNA biogenesis, mechanisms of actions, and circulation.

2. Shenoy A, Blelloch RH. Regulation of microRNA function in somatic stem cell proliferation and differentiation. Nat. Rev. Mol. Cell. Biol. 15(9), 565-576 (2014).

3. Rottiers V, Näär AM. MicroRNAs in metabolism and metabolic disorders. Nat. Rev. Mol. Cell. Biol. 13(4), 239-250 (2012).

4. Jovanovic M, Hengartner MO. miRNAs and apoptosis: RNAs to die for. Oncogene 25(46), 6176-6187 (2006).

5. O'Connell RM, Rao DS, Baltimore D. microRNA regulation of inflammatory responses. Annu. Rev. Immunol. 30 295-312 (2012).

6. Wang J, Chen J, Sen S. MicroRNA as biomarkers and diagnostics. J. Cell. Physiol. 231(1), 25-30 (2016)

7. Moldovan L, Batte KE, Trgovcich J, Wisler J, Marsh CB, as circulating biomarkers. J. Cell. Mol. Med. 18(3), 371-390 (2014).

8. Dellett $M$, Simpson DA. Considerations for optimization of microRNA PCR assays for molecular diagnosis. Expert. Rev. Mol. Diagn. 16(4), 407-414 (2016).

9. Hunt EA, Broyles D, Head T, Deo SK. MicroRNA detection: current technology and research strategies. Annu. Rev. Anal. Chem. 8, 217-237 (2015).

10. Dard-Dascot C, Naquin D, d'Aubenton-Carafa Y, Alix K, RNA library preparation protocols for next-generation sequencing. BMC Genomics 19(1), 118 (2018).

11. Wang B, Xi Y. Challenges for microRNA microarray data analysis. Microarrays 2(2), 34 (2013). Specific for Precursor and Mature miRNAs. Humana Press, NJ, USA, 121-124 (2014).

13. Lao K, Xu NL, Yeung V, Chen C, Livak KJ, Straus NA. Front. Endocrinol. 9, 402 (2018). Piper M. Methodological challenges in utilizing miRNA Thermes C, van Dijk E. Systematic comparison of smal

12. Zöllner H, Hahn SA, Maghnouj A. Quantitative RT-PCR
Multiplexing RT-PCR for the detection of multiple miRNA species in small samples. Biochem. Biophys. Res. Commun. 343(1), 85-89 (2006).

14. Kappel A, Keller A. miRNA assays in the clinical laboratory: workflow, detection technologies and automation aspects. Clin. Chem. Lab. Med. 55(5), 636-647 (2017).

15. Schmittgen T, Lee E, Jiang J, Sarkar A. Real-time PCR quantification of precursor and mature microRNA. Methods 44(1), 31-38 (2008).

16. Fiedler SD, Carletti MZ, Christenson LK. Quantitative RT-PCR methods for mature microRNA expression anatysis. In: Methods in Molecular Biology. King N. Humana Press, NJ, USA, 49-64 (2010).

17. Shi R, Chiang VL. Facile means for quantifying microRNA expression by real-time PCR. Biotechniques 39(4), 519-525 (2005) Sensitive and specific miRNA detection method using SplintR Ligase. Nucleic Acids Res. 44(13), 116 (2016).

19. Kappel $A$, Backes $C$, Huang $Y$ et al. MicroRNA in Chem. 61(4), 600-607 (2015).

20. Munafó DB, Robb GB. Optimization of enzymatic reaction conditions for generating representative pools of cDNA from small RNA. RNA 16(12), 2537-2552 (2010).

21. Pfaffl MW, Hageleit M. Validities of mRNA quantification using recombinant RNA and recombinant DNA external calibration curves in real-time RT-PCR. Biotechnol. Lett. 23(4), 275-282 (2001).

22. Bustin SA, Benes V, Garson JA et al. The MIQE guidelines: minimum information for publication of quantitative real-time PCR experiments. Clin. Chem. 55(4), 611-622 (2009)

23. Bustin SA. Quantification of mRNA using real-time reverse transcription PCR (RT-PCR): trends and problems. J. Mol. Endocrinol. 29, 23-39 (2002).

24. Vychytilova-Faltejskova $P$, Radova L, Sachlova M et al.
18. Jin J, Vaud S, Zhelkovsky AM, Posfai J, McReynolds LA vitro diagnostics using immunoassay analyzers. Clin.

Serum-based microRNA signatures in early diagnosis and prognosis prediction of colon cancer. Carcinogene sis 37(10), 941-950 (2016).

25. Androvic $P$, Valihrach L, Elling J, Sjoback R, Kubista $M$. Two-tailed RT-qPCR: a novel method for highly accurat miRNA quantification. Nucleic Acids Res. 45(15), 144 (2017).

26. Leshkowitz D, Horn-Saban S, Parmet Y, Feldmesser E. Differences in microRNA detection levels are technology and sequence dependent. RNA 19(4), 527-538 (2013).

27. Stahlberg A, Håkansson J, Xian X, Semb H, Kubista M. Properties of the reverse transcription reaction in mRNA quantification. Clin. Chem. 50(3), 509-515 (2004).

28. Miranda JA, Steward GF. Variables influencing the efficiency and interpretation of reverse transcription quantitative PCR (RT-qPCR): an empirical study using bacteriophage MS2. J. Virol. Methods. 241, 1-10 (2017)

29. Redshaw N, Wilkes T, Whale A, Cowen S, Huggett J, Foy CA. A comparison of miRNA isolation and RT-qPCR technologies and their effects on quantification accuracy and repeatability. Biotechniques 54(3), 155-164 (2013).

30. Wang J, Jiang D, Rao $\mathrm{H}$, Zhao J, Wang $Y$, Wei L. Absolute quantification of serum microRNA-122 and its correlaquantification of serum quanthe aminotransferase in chronic Infect. Dis. 30, 52-56 (2015).

31. Iguchi T, Niino N, Tamai S, Sakurai K, Mori K. Absolute Quantification of plasma microRNA levels in Cynomolgus monkeys, using quantitative real-time reverse transcription PCR. J. Vis. Exp. e56850 (2018).

32. Bustin SA, Nolan T. Pitfalls of quantitative real-time reverse-transcription polymerase chain reaction. $J$. Biomol. Tech. 15(3), 155-166 (2004).

33. Huggett J, Dheda K, Bustin S, Zumla A. Real-time RT-PCR normalisation; strategies and considerations. Genes. Immun. 6(4), 279-284 (2005).

34. Curry J, McHale C, Smith MT. Low efficiency of the moloney murine leukemia virus reverse transcriptase during reverse transcription of rare t $(8 ; 21)$ fusion gene transcripts. Biotechniques 32(4), gene transcripts.

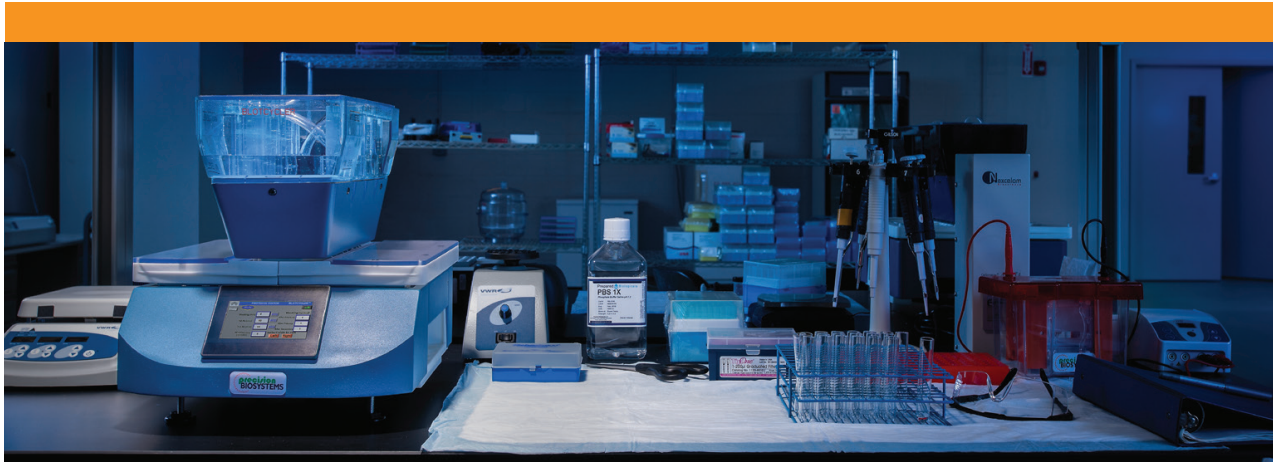

\section{How to control variability in your Western blot assays:}

By eliminating manual errors in fluid delivery, timing and washing, Blot Cycler $^{\text {Tw }}$ consistently delivers reproducible Western blot results. BlotCycler performs every step in the processing protocol precisely, delivering an

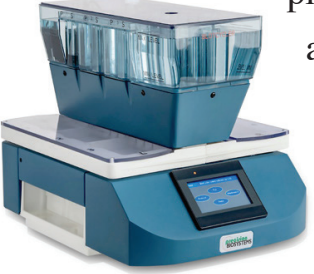
affordable solution to busy labs that demand productivity.

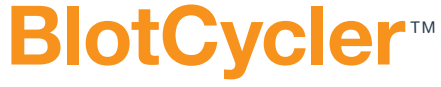

bY PRECISION BIOSYSTEMS

35. Chen C, Ridzon DA, Broomer AJ et al. Real-time quantification of microRNAs by stem-loop RT-PCR. Nucleic Acids Res. 33(20), 179 (2005).

36. Liu Y, Chen S, Zhang J et al. Analysis of serum microRNAs as potential biomarker in coronary bifurcation lesion. Dis. Markers 2015, 1-5 (2015).

37. Rainer TH, Leung LY, Chan CPY et al. Plasma miR-124-3p and miR-16 concentrations as prognostic markers in acute stroke. Clin. Biochem. 49(9), 663-668 (2016)

38. Nagaraj S, Laskowska-Kaszub K, Debsk $\mathrm{KJ}$ et al. Profile of 6 microRNA in blood plasma distinguish early stage Alzheim er disease patients from non-demented subjects. Oncotarget 8(10), 1612216143 (2017). 